\title{
Induction of labour versus expectant management for large-for-date fetuses: a randomised controlled trial
}

\author{
Michel Boulvain, Marie-Victoire Senat, Franck Perrotin, Norbert Winer, Gael Beucher, Damien Subtil, Florence Bretelle, Elie Azria, \\ Dominique Hejaiej, Françoise Vendittelli, Marianne Capelle, Bruno Langer, Richard Matis, Laure Connan, Philippe Gillard, Christine Kirkpatrick, \\ Gilles Ceysens, Gilles Faron, Olivier Irion, Patrick Rozenberg, for the Groupe de Recherche en Obstétrique et Gynécologie (GROG)
}

\section{Summary}

Background Macrosomic fetuses are at increased risk of shoulder dystocia. We aimed to compare induction of labour with expectant management for large-for-date fetuses for prevention of shoulder dystocia and other neonatal and maternal morbidity associated with macrosomia.

Methods We did this pragmatic, randomised controlled trial between Oct 1, 2002, and Jan 1, 2009, in 19 tertiary-care centres in France, Switzerland, and Belgium. Women with singleton fetuses whose estimated weight exceeded the 95th percentile, were randomly assigned (1:1), via computer-generated permuted-block randomisation (block size of four to eight) to receive induction of labour within 3 days between $37^{+0}$ weeks and $38^{+6}$ weeks of gestation, or expectant management. Randomisation was stratified by centre. Participants and caregivers were not masked to group assignment. Our primary outcome was a composite of clinically significant shoulder dystocia, fracture of the clavicle, brachial plexus injury, intracranial haemorrhage, or death. We did analyses by intention to treat. This trial is registered with ClinicalTrials.gov, number NCT00190320.

Findings We randomly assigned 409 women to the induction group and 413 women to the expectant management group, of whom 407 women and 411 women, respectively, were included in the final analysis. Mean birthweight was $3831 \mathrm{~g}$ (SD 324) in the induction group and $4118 \mathrm{~g}$ (392) in the expectant group. Induction of labour significantly reduced the risk of shoulder dystocia or associated morbidity $(n=8)$ compared with expectant management $(n=25$; relative risk $[R R] 0.32,95 \%$ CI $0.15-0.71 ; p=0.004)$. We recorded no brachial plexus injuries, intracranial haemorrhages, or perinatal deaths. The likelihood of spontaneous vaginal delivery was higher in women in the induction group than in those in the expectant management group (RR 1·14, 95\% CI 1·01-1·29). Caesarean delivery and neonatal morbidity did not differ significantly between the groups.

Interpretation Induction of labour for suspected large-for-date fetuses is associated with a reduced risk of shoulder dystocia and associated morbidity compared with expectant management. Induction of labour does not increase the risk of caesarean delivery and improves the likelihood of spontaneous vaginal delivery. These benefits should be balanced with the effects of early-term induction of labour.

Funding Assistance Publique-Hôpitaux de Paris and the University of Geneva.

\section{Introduction}

Macrosomia is a risk factor for unfavourable delivery outcomes, including operative vaginal or caesarean delivery and shoulder dystocia., ${ }^{1,2}$ Shoulder dystocia can cause neonatal morbidity, including fracture of the clavicle, brachial plexus injury, or asphyxia. Elective caesarean section can be done to avoid a vaginal delivery complicated by macrosomia. However, findings from a decision analysis ${ }^{3}$ suggested that the number of elective caesarean sections needed to avoid one permanent brachial plexus injury is quite high. This strategy is thus recommended only when fetal weight is estimated to exceed $4500 \mathrm{~g}$ for women with diabetes and $5000 \mathrm{~g}$ for those without diabetes. ${ }^{4}$

Another option would be to induce labour, which reduces the opportunity for continued fetal growth and, theoretically, decreases the risk of caesarean section for cephalopelvic disproportion, and reduces the risk of operative vaginal delivery, perineal trauma, and shoulder dystocia. Nonetheless, induction of labour can fail, which would make caesarean delivery necessary. Early-term (37-38 weeks) delivery, especially by elective caesarean section, might also increase the risk of mortality and morbidity of the neonate, including long-term development issues. ${ }^{5,6}$

Several investigators have raised questions about induction of labour for macrosomic fetuses, especially because most observational studies have associated this strategy with an increased risk of caesarean delivery, with no significant decrease in shoulder dystocia. ${ }^{7}$ A systematic review, ${ }^{8}$ which included the few randomised trials published, ${ }^{9,10}$ showed no difference in the risk of caesarean section between the labour induction and expectant management groups, but also no benefit of labour induction in prevention of neonatal trauma. The conclusions were limited by the relatively small sample size of the trials and by the inclusion of women, usually at 40 weeks of gestation or more, carrying a fetus with an
Published Online April 9, 2015 http://dx.doi.org/10.1016/ S0140-6736(14)61904-8

See Online/Comment http://dx.doi.org/10.1016 S0140-6736(14)62302-3 Département de Gynécologie et d'Obstétrique, Geneva University Hospitals and Faculty of Medicine, University of Geneva, Geneva, Switzerland (Prof M Boulvain MD, Prof O Irion MD); Département de Gynécologie-Obstétrique, APHP, Hôpital Bicêtre, Hôpita Antoine Béclère, Université Paris Sud, Faculté de Medecine, Orsay, Paris, France (Prof M-V Senat MD); Pôle de Gynécologie-Obstétrique, Hôpital Bretonneau, CHRU Tours, Tours, France (Prof F Perrotin MD) Département de GynécologieObstétrique, Hôpital MèreEnfant, Nantes, France (N Winer MD); Département de Gynécologie-Obstétrique et Médecine de la Reproduction, CHU de Caen, Caen, France (G Beucher MD); Département de Gynécologie-Obstétrique, Hôpital Jeanne de Flandre, Lille, France (Prof D Subtil MD); Département de GynécologieObstétrique, Hôpital Nord, Marseille, France (Prof F Bretelle MD); Département de GynécologieObstétrique, Hôpital Bichat, AP-HP, Paris, France

(E Azria MD); Département de Gynécologie-Obstétrique, Centre Hospitalier Régional, Annecy, France (D Hejaiej MD); Pôle de GynécologieObstétrique et Reproduction Humaine, CHU de ClermontFerrand, Hôpital Estaing, Clermont-Ferrand, France (F Vendittelli MD); Département de Gynécologie-Obstétrique, Hôpital de La Conception, Marseille, France (M Capelle MD); Département de Gynécologie-Obstétrique, Hôpital Hautepierre, 
Strasbourg, France (Prof B Langer MD); Groupe Hospitalier de I'Institut Catholique de Lille, Lille, France (R Matis MD); Département de Gynécologie-Obstétrique, Hôpital Paul de Viguier, Toulouse, France (L Connan MD); Pôle de Gynécologie-Obstétrique, Hôpital Hôtel Dieu, Angers,

France (P Gillard MD); Département de GynécologieObstétrique, Hôpital Erasme, Bruxelles, Belgium (C Kirkpatrick MD, G Ceysens MD); Département de Gynécologie-Obstétrique, Hôpital Ambroise Paré, Mons, Belgium (G Ceysens); Département de GynécologieObstétrique, Hôpital Brugmann, Bruxelles, Belgium (G Faron MD); Département de Gynécologie-Obstétrique, Hôpital Poissy Saint-Germain, Université VersaillesSt Quentin, France (Prof P Rozenberg MD)

Correspondence to: Prof Michel Boulvain, MaternitéHUG, Geneva CH-1211, Switzerland michel.boulvain@hcuge.ch estimated weight of more than $4000 \mathrm{~g}$. Inclusion of these women is likely to restrict the benefit of induction of labour, because this intervention at that stage of gestation leads to very small differences between induced labour and expectant management groups, in both mean gestational age at birth and birthweight.

We assessed the risks and benefits of induction of labour compared with expectant management in women with large-for-date fetuses. We postulated that induction of labour would prevent shoulder dystocia and other neonatal and maternal morbidity associated with macrosomia, with no major changes in the risk of caesarean section.

\section{Methods \\ Study design and participants}

We did this multicentre, randomised controlled trial in 19 tertiary-care university hospitals in France, Switzerland, and Belgium. Recruitment started on Oct 1, 2002, in four hospitals and was extended to France in 2005, which added 15 more hospitals. Recruitment ended on Jan 1, 2009.

Eligible women had a singleton macrosomic fetus in cephalic presentation and no contraindications to planned vaginal delivery. We identified the women in two stages, between 36 weeks and 38 weeks of gestation, during routine antenatal care visits. First, we screened for large-for-date fetuses (weighing more than the 90th percentile), on the basis of either fundal height or fetal weight estimated with the Leopold manoeuvres. Then, if the fetus was estimated to weigh more than the 90th percentile, we estimated fetal weight sonographically with Hadlock's formula. ${ }^{11}$ We included women if the estimated weight of the fetus was more than the

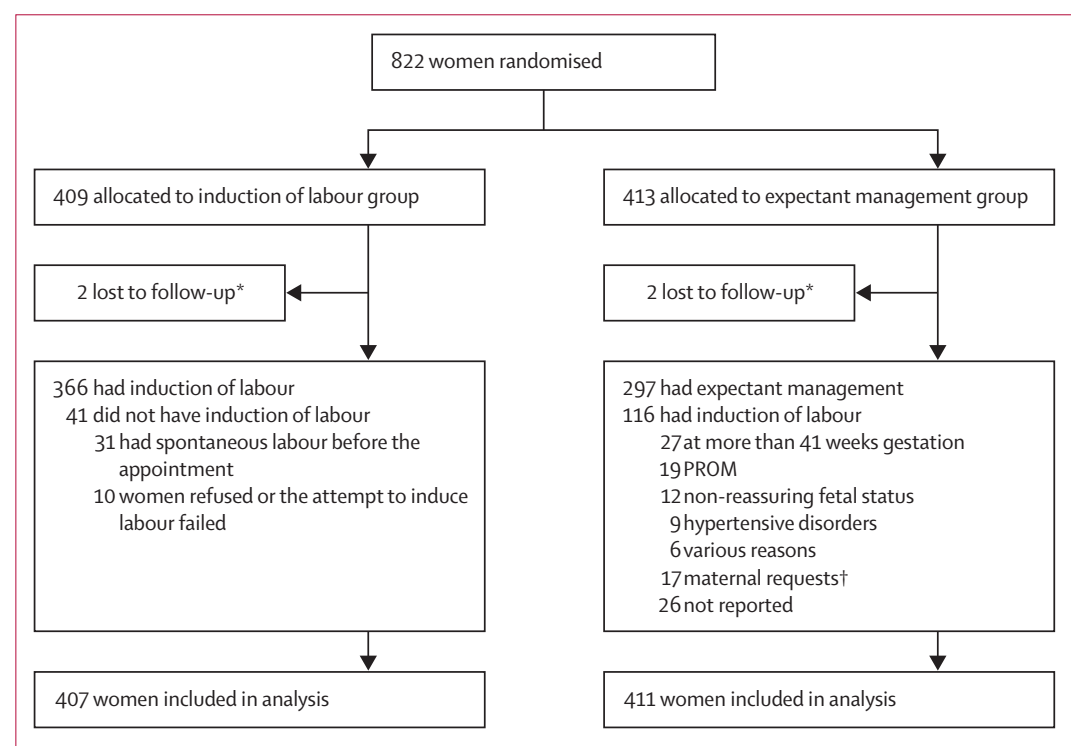

Figure: Trial profile

*Participants were lost to follow-up before delivery, so had no data for assessment of the measurement outcomes. tIncluded fear of delivery of a large neonate. 95th percentile ( $3500 \mathrm{~g}$ at 36 weeks of gestation, $3700 \mathrm{~g}$ at 37 weeks, and $3900 \mathrm{~g}$ at 38 weeks).

Exclusion criteria were any contraindication to induction of labour or vaginal delivery, history of caesarean section, neonatal trauma or shoulder dystocia, severe urinary or faecal incontinence, and insulin-treated diabetes.

We based gestational age on last menstrual periods. If the sonography, routinely done in all centres during the first trimester, showed a discrepancy of more than 5 days, we used the sonographic date.

The study protocol was approved for all centres in France by the Ethics Committee of the Poissy SaintGermain Hospital (Comité de Protection des Personnes), in Saint-Germain en Laye. The protocol was approved by the institutional ethics committees in Switzerland and Belgium. All women provided written informed consent.

\section{Randomisation and masking}

Women were randomly assigned (1:1), via centralised computer-generated randomisation with permuted blocks (block size of four to eight), to receive induction of labour or expectant management. Randomisation was stratified by centre. Clinicians and participants had no access to the list, but were not masked to group allocation, which was made known after entry of the women, screening, and confirmation of consent. Investigators were masked only in the assessment of uncertain primary outcome. The decision about the nonsignificance of shoulder dystocia in these cases was made by investigators masked to the group allocation.

\section{Procedures}

We induced labour between $37^{+0}$ weeks and $38^{+6}$ and within 3 days after randomisation. The attending physician chose the method for cervical ripening and labour induction, according to local practice. Women with an unfavourable cervix had cervical ripening with prostaglandin E2 or misoprostol. Oxytocin was then used to induce uterine contractions, if labour did not start during ripening. Expectant management continued until either spontaneous labour or diagnosis of a condition necessitating induction according to the hospital's policy (eg, pregnancy continuing beyond 41 weeks of gestation, premature rupture of membranes).

\section{Outcomes}

The primary outcome was a composite of significant shoulder dystocia, fracture of the clavicle or a long bone, brachial plexus injury, intracranial haemorrhage, or death. We defined clinically significant shoulder dystocia as difficulty with delivery of the shoulders that was not resolved by the McRoberts' manoeuvre (flexion of the maternal thighs), usually combined with suprapubic pressure. Manoeuvres whose use suggested significant shoulder dystocia were those involving rotation of the fetus to displace the anterior shoulder impacted behind the maternal pubic bone (Woods, Rubin, or Jacquemier 
manoeuvres)..$^{12}$ The definition also included births with an interval of $60 \mathrm{~s}$ or more between delivery of the head and the body. ${ }^{13}$

Our prespecified secondary outcomes were: maternal morbidity, defined as caesarean section, operative vaginal delivery (vacuum or forceps), postpartum haemorrhage (1000 mL or more), blood transfusion, and anal sphincter tear; and neonatal morbidity, defined as arterial cord blood $\mathrm{pH}$ less than 7.10, Apgar score at 5 min less than 7 , and admission to the neonatal intensive-care unit. We also obtained information about other outcomes, including concentrations of blood bilirubin. We defined clinically significant hyperbilirubinaemia as a maximum value exceeding $350 \mathrm{mmol} / \mathrm{L}$.

\section{Statistical analysis}

Analysis was by intent to treat. We report baseline characteristics and outcomes as means (SDs), medians (IQRs), or numbers and percentages. We report the effects of the intervention on outcomes as relative risks (RRs), risk differences, and numbers needed to treat, with 95\% CIs. Stratified analysis with the MantelHaenszel method enabled adjustment of the RR estimate for parity (primiparity and multiparity), obesity (bodymass index $\leq 30 \mathrm{~kg} / \mathrm{m}^{2}$ and $>30 \mathrm{~kg} / \mathrm{m}^{2}$ ), and centre. We tested significance with Fisher's exact test. We did analysis with SPSS (versions 18 and 20).

We based the initial sample size calculation on detection of a difference in percentages of the primary outcome, with a power of $80 \%$ and a type 1 error of $5 \%$. We assumed the risk in the control group to be $5-10 \%$ and the risk in the induction of labour group to be $1 \cdot 65-5 \cdot 00 \%$ (ie, an RR of $0 \cdot 33-0 \cdot 50$ ). The calculation showed that a total sample size of about 1000 women (500 per group) was sufficient to show these differences. Financial constraints made it necessary to end recruitment at a predetermined date (Jan 1, 2009), before we did any analyses.

The study is registered with ClinicalTrials.gov, number NCT00190320.

\section{Role of the funding source}

The funder of the study had no role in study design, data collection, data analysis, data interpretation, or writing of the report. MB had full access to all the data in the study and $\mathrm{MB}$ and $\mathrm{PR}$ had final responsibility for the decision to submit for publication.

\section{Results}

The figure shows the trial profile. We randomly assigned 822 women to the induction of labour group $(n=409)$ or the expectant management group $(n=413)$. Four $(1 \%)$ women were lost to follow-up before delivery, leaving 818 women in the final analysis. Labour was induced in $366(89 \%)$ women in the induction group and $116(28 \%)$ women in the expectant management group (figure). Baseline characteristics were similar between groups (table 1).
Mean birthweight was $3831 \mathrm{~g}$ (324) in the induction group and $4118 \mathrm{~g}$ (392) in the expectant group. 125 neonates had a birthweight of $4000 \mathrm{~g}$ or more and 13 neonates weighed $4500 \mathrm{~g}$ or more in the induction group, compared with

\begin{tabular}{|c|c|c|}
\hline & $\begin{array}{l}\text { Induction of labour group } \\
(n=407)\end{array}$ & $\begin{array}{l}\text { Expectant management } \\
\text { group }(n=411)\end{array}$ \\
\hline Maternal age (years) & $29 \cdot 2(5 \cdot 3)$ & $29 \cdot 8(5 \cdot 3)$ \\
\hline BMI before pregnancy $\left(\mathrm{kg} / \mathrm{m}^{2}\right)$ & $26 \cdot 1(5 \cdot 7)$ & $25 \cdot 6(5 \cdot 4)$ \\
\hline Weight gain $(\mathrm{kg})$ & $14 \cdot 7(6 \cdot 2)$ & $15 \cdot 6(6 \cdot 6)$ \\
\hline \multicolumn{3}{|c|}{ Gestational age at randomisation (weeks) } \\
\hline $36^{+0}$ to $\leq 37$ & $42(10 \%)$ & $44(11 \%)$ \\
\hline 37 to $\leq 38$ & $177(44 \%)$ & $181(44 \%)$ \\
\hline 38 to $\leq 39$ & $187(46 \%)$ & $184(45 \%)$ \\
\hline Nulliparity & $191(47 \%)$ & $208(51 \%)$ \\
\hline Previous history of macrosomia* ${ }^{*}$ & $65 / 212(31 \%)$ & $62 / 200(31 \%)$ \\
\hline Gestational diabetes $\neq$ & $39(10 \%)$ & $43(11 \%)$ \\
\hline Fundal height (cm) & $36 \cdot 3(2 \cdot 3)$ & $36 \cdot 3(2 \cdot 4)$ \\
\hline Estimated weight (g, clinical) & $3850(297)$ & $3901(296)$ \\
\hline Estimated weight (g, sonography) & 3964 (229) & $3971(238)$ \\
\hline Male fetus & $232(57 \%)$ & $236(57 \%)$ \\
\hline \multicolumn{3}{|c|}{$\begin{array}{l}\text { Numbers are mean (SD), } \mathrm{n}(\%) \text {, or } \mathrm{n} / \mathrm{N}(\%) \text {. *In multiparous women. †Some information missing. 抽eated with } \\
\text { diet only. }\end{array}$} \\
\hline
\end{tabular}

\begin{tabular}{|c|c|c|c|}
\hline & $\begin{array}{l}\text { Induction of } \\
\text { labour group } \\
(n=407)\end{array}$ & $\begin{array}{l}\text { Expectant } \\
\text { management } \\
\text { group }(n=411)\end{array}$ & $\begin{array}{l}\mathrm{RR}(95 \% \mathrm{Cl}) \\
\text { or p value }\end{array}$ \\
\hline Composite primary outcome & $8(2 \%)$ & $25(6 \%)$ & $0.32(0.15-0.71)$ \\
\hline Significant shoulder dystocia & $5(1 \%)$ & $16(4 \%)$ & $0.32(0.12-0.85)$ \\
\hline Delay of $\geq 60 \mathrm{~s}$ & $2(1 \%)$ & $10(2 \%)$ & $0.20(0.04-0.92)$ \\
\hline Fracture & $2(1 \%)$ & $8(2 \%)$ & $0.25(0.05-1.18)$ \\
\hline Brachial plexus injury & 0 & 0 & .. \\
\hline Intracranial haemorrhage & 0 & 0 & .. \\
\hline Death & 0 & 0 & .. \\
\hline Any shoulder dystocia & $15(4 \%)$ & $32(8 \%)$ & $0.47(0.26-0.86)$ \\
\hline \multicolumn{4}{|l|}{ Mode of delivery } \\
\hline Spontaneous vaginal & $239(59 \%)$ & $212(52 \%)$ & $1 \cdot 14(1 \cdot 01-1 \cdot 29)$ \\
\hline Forceps or vacuum & $54(13 \%)$ & $68(17 \%)$ & $0.80(0.58-1.12)$ \\
\hline Caesarean section & $114(28 \%)$ & $130(32 \%)$ & $0.89(0.72-1.09)$ \\
\hline Perineal tear (episiotomy or second degree) & $148(36 \%)$ & $158(38 \%)$ & $0.95(0.79-1.13)$ \\
\hline Anal sphincter tear & $6(2 \%)$ & $2(1 \%)$ & $3.03(0.62-14.92)$ \\
\hline Vaginal laceration or cervical tear & $5(1 \%)$ & $1(<1 \%)$ & $5.05(0.59-43.02)$ \\
\hline Blood transfusion & $4(1 \%)$ & $3(1 \%)$ & $1.35(0.30-5.98)$ \\
\hline Haemorrhage ( $\geq 1000 \mathrm{~mL}$ ) & $12(3 \%)$ & $21(5 \%)$ & $0.58(0.29-1.16)$ \\
\hline Retained placenta & $3(1 \%)$ & $4(1 \%)$ & $0.76(0.17-3.36)$ \\
\hline Sepsis & $1(<1 \%)$ & $1(<1 \%)$ & $1.01(0.06-16.1)$ \\
\hline Fever $\left(>38.5^{\circ} \mathrm{C}\right)$ & $3(1 \%)$ & $6(2 \%)$ & $0.58(0.29-1.16)$ \\
\hline \multicolumn{4}{|l|}{ Duration of hospital stay } \\
\hline Before delivery (h) & $16 \cdot 2(8 \cdot 1-31 \cdot 4)$ & $7 \cdot 6(4 \cdot 6-11 \cdot 6)$ & $p<0.0001$ \\
\hline After delivery (days) & $4 \cdot 0(4 \cdot 0-5 \cdot 0)$ & $4 \cdot 0(4 \cdot 0-5 \cdot 0)$ & $p=0.61$ \\
\hline
\end{tabular}




\begin{tabular}{|c|c|c|c|}
\hline & $\begin{array}{l}\text { Induction of } \\
\text { labour group } \\
(n=407)\end{array}$ & $\begin{array}{l}\text { Expectant } \\
\text { management } \\
\text { group }(n=411)\end{array}$ & p value \\
\hline Apgar score $<7$ at 5 min & $3(1 \%)$ & $2(1 \%)$ & 0.99 \\
\hline \multicolumn{4}{|l|}{ Cord blood pH } \\
\hline$<7 \cdot 10$ & $12(3 \%)$ & $12(3 \%)$ & 1.00 \\
\hline$<7.00$ & $1(<1 \%)$ & $1(<1 \%)$ & 1.00 \\
\hline \multicolumn{4}{|l|}{ Highest bilirubin concentration ( $\mathrm{mmol} / \mathrm{L}$ ) } \\
\hline$>250$ & $36(9 \%)$ & $12(3 \%)$ & 0.0004 \\
\hline$>350$ & 0 & 0 & 1.00 \\
\hline Phototherapy & $45(11 \%)$ & $27(7 \%)$ & 0.03 \\
\hline Hypoglycaemia & $9(2 \%)$ & $13(3 \%)$ & 0.40 \\
\hline Admission to neonatal intensive care unit & $15(4 \%)$ & $23(6 \%)$ & 0.19 \\
\hline Transient tachypnoea of the newborn (wet lung) & $1(<1 \%)$ & $1(<1 \%)$ & 1.00 \\
\hline Use of CPAP & $2(1 \%)$ & $1(<1 \%)$ & 0.99 \\
\hline
\end{tabular}

254 neonates who weighed $4000 \mathrm{~g}$ or more and 61 who weighed $4500 \mathrm{~g}$ or more in the expectant group. The mean difference in time between randomisation and delivery was of 4.9 days $(4 \cdot 1)$ in the induction group and and $15 \cdot 4$ days $(8 \cdot 4)$ in the expectant management group.

Table 2 shows the number of occurrences of the elements of the composite primary outcome measure. We recorded the primary outcome in eight $(2 \%)$ of 407 deliveries in the induction group and $25(6 \%)$ of 411 deliveries in the control group (table $2 ; \mathrm{p}=0 \cdot 004$ ). The risk difference was $4 \%(95 \%$ CI $1.4-6 \cdot 8)$ and the number needed to treat was 25 (95\% CI 15-70). The estimated benefit did not change when the definition of the primary outcome excluded the interval of $60 \mathrm{~s}$ or more between delivery of the head and body (RR $0 \cdot 34,95 \%$ CI $0 \cdot 14-0 \cdot 78$ ). No brachial plexus injuries, intracranial haemorrhage, or perinatal deaths were recorded. The main outcome occurred in three neonates in the induction group and four in the expectant management group in the neonates weighing less than $4000 \mathrm{~g}$. In neonates with birthweights of $4000 \mathrm{~g}$ or more, we noted the primary outcome in five neonates in the induction group and 21 in the expectant management group. The RRs adjusted for parity $(0 \cdot 32$, 95\% CI $0 \cdot 15-0 \cdot 71$ ), obesity (RR $0 \cdot 32$, 95\% CI $0 \cdot 15-0 \cdot 71$ ), and centre (RR $0 \cdot 31,95 \%$ CI $0 \cdot 14-0 \cdot 72$ ) did not differ from the crude RR.

The incidence of caesarean section and operative vaginal delivery did not differ significantly between the groups (table 2). The likelihood of spontaneous vaginal delivery increased significantly in the induction of labour group (table 2). Anal sphincter tears were infrequent and did not differ significantly between groups (table 2). Postpartum haemorrhage of more than $1000 \mathrm{~mL}$ happened in 12 (3\%) women in the induction group and $21(5 \%)$ women in the expectant management group (table 2).

The prespecified neonatal outcomes did not differ significantly between groups (table 3 ). The number of neonates admitted to the neonatal intensive-care unit (15 [4\%] in the induction group and 23 [6\%] the expectant management group) was similar in each group, and each group included one baby with transient tachypnoea. No neonates in either group had hyperbilirubinaemia exceeding $350 \mathrm{mmol} / \mathrm{L}$, although when we lowered the cut-off to $250 \mathrm{mmol} / \mathrm{L}$, incidence was higher in the induction group (table 3). Phototherapy was used more often for neonates in the induction group (table 3), especially for those randomised to induction of labour before 38 weeks of gestation (28 [13\%] of 219 neonates in the induction group vs 16 [7\%] of 225 neonates in the expectant group; randomisation at 38 weeks: 17 [9\%] of 187 neonates in the induction group vs 11 [6\%] of 185 in the expectant group).

\section{Discussion}

Our findings show that induction of labour for large-fordate fetuses significantly reduces the risks of shoulder dystocia and bone fracture, and increases the likelihood of spontaneous vaginal delivery. We recorded no brachial plexus injuries, intracranial haemorrhages, or perinatal deaths, nor did we detect differences between the groups for markers of asphyxia at birth.

Our results differ from those of a systematic review, which reported an increased risk of caesarean delivery with no decrease in the risk of neonatal trauma. These previous results form the basis of present guidelines that advise against induction of labour to prevent macrosomia. $^{4,14}$ In 2012, the results of a very large database study ${ }^{15}$ showed that women with babies weighing more than $4000 \mathrm{~g}$ induced at 39 weeks had fewer caesarean sections than women whose labour was either induced or spontaneous at 40 weeks or later. An important limitation, however, is that its analysis was according to known birthweight, rather than estimated fetal weight.

The only two published randomised trials ${ }^{9,10}$ did not show a significant benefit to the mother or child from induction of labour, although these studies had small sample sizes (273 women and 40 women) and used inclusion criteria that restricted their ability to show a difference. Women were included when the fetus was estimated to weigh more than $4000 \mathrm{~g}$, which is usually at around 40 weeks, when the daily probability of spontaneous labour is high. Labour induction was done only a few days before labour would have begun spontaneously, so the difference in birthweight between the induced labour and expectant management groups was very small $(63 \mathrm{~g})$. The benefits for prevention of shoulder dystocia and other macrosomia-associated morbidities were thus smaller than they would have been had the intervention been done earlier. Nonetheless, one of these studies ${ }^{9}$ reported a lower incidence of fetal trauma (fracture or brachial plexus injury) in the induction group, with all six cases recorded in the expectant management group. 9 An unpublished pilot 
randomised trial (ISRCTN98146741), which included 59 women, also showed no benefit for induction of labour. Our trial is larger than previous studies, with earlier inclusion and intervention and therefore greater differences in birthweight between groups and a higher probability of differences being associated with induction.

A limitation of our trial is that the sample size was smaller than initially planned. We originally planned to include 1000 women, with an interim analysis after the first 500 women. Because recruitment was slower than expected and funding was ending, we revised this plan and decided to stop recruitment on a prespecified date, before we did any analysis; the trial results did not affect the decision to stop recruitment. Moreover, although recruitment stopped early, our study has a larger sample size than previous randomised trials.

Another limitation is the absence of masking of both clinicians and women, which would be impossible in view of the nature of the intervention. This absence might have led doctors to do caesarean section in some women based on the knowledge of estimated weight, which was larger in fetuses in the expectant management group than in those in the induced labour group. An observational study ${ }^{16}$ has shown that clinicians are more prone to do a caesarean section when macrosomia is suspected than when it is not.

Any strategy to detect macrosomic fetuses is limited by the imprecision of the methods for estimation of fetal weight. ${ }^{17}$ Fundal height is imprecise, subject to measurement errors, and dependent on the thickness of the maternal abdominal wall and the amount of amniotic fluid. ${ }^{18}$ Ultrasound is also imprecise in estimation of fetal weight, especially for large-for-date fetuses..$^{19}$ However, our two-step procedure was sufficiently reliable for screening of large fetuses that might benefit from induction of labour. Restriction of sonographic estimation of the fetal weight to fetuses regarded as clinically large ensured that the number of scans needed was not unduly increased.

The components of the composite primary outcome that we noted in our study-clinically significant shoulder dystocia and bone fractures-are unfavourable for women and babies, and for clinicians are among the most frequent causes of litigation and damage awards..$^{20}$ Fortunately, we recorded no instances of permanent brachial plexus injury or death, which are at the severe end of the composite primary outcome components. A trial to assess the advantages of earlier induction of labour for these two events would probably be impossible to do because of their very low occurrence (less than $10 \%$ in neonates with shoulder dystocia); we estimate a sample size of 7800 would be needed to show a difference between $0.6 \%$ and $0.2 \%$. However, we detected a difference between the groups for all components of our primary outcome, and the magnitude of the benefit increased with the severity of the definition. We therefore postulate that induction of labour might prevent shoulder dystocia that is associated with permanent brachial plexus injury or death.

\section{Panel: Research in context}

\section{Systematic review}

Fetal macrosomia is a risk factor for a complicated delivery. Neonates with increased birthweight have an increased risk of shoulder dystocia and associated morbidities (fracture, brachial plexus injury, and asphyxia) whereas the mother is at risk for caesarean section, instrumental delivery, and perineal tears. This issue is complicated by the inaccuracy of the various methods to estimate fetal weight. We searched Medline and the Cochrane Library from inception to July 1, 2014 with the search terms "induction of labour", "macrosomia", "shoulder dystocia", and "randomised", according to the strategy of the Cochrane Collaboration. Our search identified two published randomised trial $\mathrm{s}^{9,10}$ and one unpublished trial (ISRCTN98146741) comparing induction of labour with expectant management for women with a fetus estimated to be large for date or macrosomic. The meta-analysis of these trials $^{8}$ showed no benefit associated with induction of labour.

\section{Interpretation}

Our results show that a policy of labour induction at 37-38 weeks of gestation for women with large-for-date fetuses reduces the risk of clinically significant shoulder dystocia or bone fracture at birth, without increasing the risk of caesarean section. These benefits should be balanced with the effects of early-term induction of labour.

The primary outcome did not include the less severe forms of shoulder dystocia that can be resolved by the McRoberts manoeuvre, because their assessment is often subjective. Additionally, in some settings, this manoeuvre is done routinely, despite the absence of evidence that it prevents shoulder dystocia in suspected macrosomia. ${ }^{21}$ Accordingly, we do not believe that use of the McRoberts manoeuvre in cases of suspected macrosomia represents a real complication of childbirth. Most cases of shoulder dystocia were in neonates with a birthweight of $4000 \mathrm{~g}$ or more, which concurs with the fact that birthweight is a risk factor, and suggests that induction of labour reduces the occurrence of this outcome by reducing birthweight.

Our definition of the primary outcome included an interval of $60 \mathrm{~s}$ or more between delivery of the head and body, which has been suggested to be an objective definition of shoulder dystocia that should reduce the risk of detection bias..$^{13}$ Some obstetricians might question the importance of this delay, because they already wait for spontaneous delivery of the shoulders after the delivery of the head of the fetus. However, exclusion of this component of the composite outcome did not change the estimate of the effect. By excluding the McRoberts manoeuvre and attempting to use objective criteria to define our primary outcome, we aimed to reduce the risk of both detection and performance bias caused by the absence of masking. However, we recorded no cases of brachial plexus injury or death and do not think that we can fairly claim to have shown more than a significant reduction in surrogate outcomes.

Despite the benefits of early induction of labour in prevention of shoulder dystocia and fracture, a policy of this procedure raises questions. The best gestational age for delivery remains controversial, because morbidity is associated with all interventions that pre-empt spontaneous 
labour. ${ }^{22}$ A large before and after study ${ }^{23}$ showed that a policy that restricts both induction of labour and elective caesarean section before 39 weeks of gestation is associated with reduced risk of admission to the neonatal intensive care unit, but an increased risk of stillbirth. We did not detect an increased risk of admission to the neonatal intensive-care unit or transient tachypnoea of the newborn, although the absence of an increase in risk of transient tachypnoea might have been because most women in our study experienced labour, rather than caesarean delivery before the onset of labour. ${ }^{24}$ Induction of labour was associated with hyperbilirubinaemia. Most neonates had phototherapy for fairly low concentrations of bilirubin, and the bilirubin concentration for which phototherapy is recommended $(350 \mathrm{mmol} / \mathrm{L})$ was never attained..$^{25}$

In summary, our findings show that induction of labour for large-for-date fetuses reduces the risk of shoulder dystocia and bone fracture, and increases the likelihood of spontaneous vaginal delivery (panel). This intervention could be offered to women with a large-fordate fetus between 37 weeks and 39 weeks of gestation.

\section{Contributors}

MB and OI did the literature search and drafted the first protocol. All authors discussed the design of the study during Groupe de Recherche en Obstétrique et Gynécologie meetings and wrote the final version of the protocol. All authors contributed to the recruitment of the participants. MB did the data analysis, with input from PR. MB and PR were responsible for writing. All authors commented on the final version of the manuscript.

\section{Declaration of interests}

We declare no competing interests.

\section{Acknowledgments}

This trial was supported by a research grant from the Département à la Recherche Clinique Ile-de-France, Assistance Publique-Hôpitaux de Paris (number PHRC AOM 04038) and by the Department of Gynecology and Obstetrics of the University of Geneva. We thank Jo Ann Cahn for doing the final editing of the manuscript; Eric Antonelli for participation in the study design; Nicole Jastrow for participation in the report writing; Riccardo Pfister for participation in the design of the neonatal data collection forms and discussions of the results for the neonate; Véronique Othenin-Girard for participation in the recruitment of participants and data collection in Geneva; and Shoreh Azimi for opening the trial's sites, verifying the data collection, and monitoring the study in France. This work was presented orally at the 32nd Congress of the Society for Maternal-Fetal Medicine (SMFM), Dallas, TX, USA, 2012

\section{References}

1 King JR, Korst LM, Miller DA, Ouzounian JG. Increased composite maternal and neonatal morbidity associated with ultrasonographically suspected fetal macrosomia. J Matern Fetal Neonatal Med 2012; 25: 1953-59.

2 Vidarsdottir H, Geirsson RT, Hardardottir H, Valdimarsdottir U, Dagbjartsson A. Obstetric and neonatal risks among extremely macrosomic babies and their mothers. Am J Obstet Gynecol 2011; 204: 423 e1-6.

3 Rouse DJ, Owen J, Goldenberg RL, Cliver SP. The effectiveness and costs of elective cesarean delivery for fetal macrosomia diagnosed by ultrasound. JAMA 1996; 276: 1480-86.

4 ACOG practice bulletin clinical management guidelines for obstetrician-gynecologists. Number 40, November 2002. Obstet Gynecol 2002; 100: 1045-50.
5 Ghartey K, Coletta J, Lizarraga L, Murphy E, Ananth CV, Gyamfi-Bannerman C. Neonatal respiratory morbidity in the early term delivery. Am J Obstet Gynecol 2012; 207: 292 e1-4.

6 De Luca R, Boulvain M, Irion O, Berner M, Pfister RE. Incidence of early neonatal mortality and morbidity after late-preterm and term cesarean delivery. Pediatrics 2009; 123: e1064-71.

7 Sanchez-Ramos L, Bernstein S, Kaunitz AM. Expectant management versus labor induction for suspected fetal macrosomia: a systematic review. Obstet Gynecol 2002; 100: 997-1002.

8 Irion O, Boulvain M. Induction of labour for suspected fetal macrosomia. Cochrane Database Syst Rev 2000; 2: CD000938.

9 Gonen O, Rosen DJ, Dolfin Z, Tepper R, Markov S, Fejgin MD. Induction of labor versus expectant management in macrosomia: a randomized study. Obstet Gynecol 1997; 89: 913-17.

10 Tey A, Eriksen N, Blanco J. A prospective randomized trial of induction versus expectant management in nondiabetic pregnancies with fetal macrosomia. Am J Obstet Gynecol 1995; 172: 293.

11 Hadlock FP, Harrist RB, Sharman RS, Deter RL, Park SK. Estimation of fetal weight with the use of head, body, and femur measurements-a prospective study. Am J Obstet Gynecol 1985; 151: 333-37.

12 Naef RW 3rd, Martin JN Jr. Emergent management of shoulder dystocia. Obstet Gynecol Clin North Am 1995; 22: 247-59.

13 Beall MH, Spong C, McKay J, Ross MG. Objective definition of shoulder dystocia: a prospective evaluation. Am J Obstet Gynecol 1998; 179: 934-37.

14 Royal College of Obstetricians and Gynaecologists. Shoulder dystocia (green-top Guideline no 42). March 28, 2012. http://www. rcog.org.uk/womens-health/clinical-guidance/shoulder-dystociagreen-top-42 (accessed April 24, 2013).

15 Cheng YW, Sparks TN, Laros RK Jr, Nicholson JM, Caughey AB. Impending macrosomia: will induction of labour modify the risk of caesarean delivery? BJOG 2012; 119: 402-09.

16 Little SE, Edlow AG, Thomas AM, Smith NA. Estimated fetal weight by ultrasound: a modifiable risk factor for cesarean delivery? Am J Obstet Gynecol 2012; 207: 309.e1-6.

17 Chauhan SP, West DJ, Scardo JA, Boyd JM, Joiner J, Hendrix NW. Antepartum detection of macrosomic fetus: clinical versus sonographic, including soft-tissue measurements. Obstet Gynecol 2000; 95: 639-42.

18 Kayem G, Grange G, Breart G, Goffinet F. Comparison of fundal height measurement and sonographically measured fetal abdominal circumference in the prediction of high and low birth weight at term. Ultrasound Obstet Gynecol 2009; 34: 566-71.

19 Simon NV, Levisky JS, Shearer DM, O’Lear MS, Flood JT. Influence of fetal growth patterns on sonographic estimation of fetal weight. J Clin Ultrasound 1987; 15: 376-83.

20 Mavroforou A, Koumantakis E, Michalodimitrakis E. Physicians' liability in obstetric and gynecology practice. Med Law 2005; 24: 1-9.

21 MacKenzie IZ, Shah M, Lean K, Dutton S, Newdick H, Tucker DE. Management of shoulder dystocia: trends in incidence and maternal and neonatal morbidity. Obstet Gynecol 2007; 110: 1059-68.

22 Clark SL, Miller DD, Belfort MA, Dildy GA, Frye DK, Meyers JA. Neonatal and maternal outcomes associated with elective term delivery. Am J Obstet Gynecol 2009; 200: 156.e1-4.

23 Ehrenthal DB, Hoffman MK, Jiang X, Ostrum G. Neonatal outcomes after implementation of guidelines limiting elective delivery before 39 weeks of gestation. Obstet Gynecol 2011; 118: $1047-55$

24 Tutdibi E, Gries K, Bucheler M, Misselwitz B, Schlosser RL, Gortner L. Impact of labor on outcomes in transient tachypnea of the newborn: population-based study. Pediatrics 2010; 125: e577-83.

25 Management of hyperbilirubinemia in the newborn infant 35 or more weeks of gestation. Pediatrics 2004; 114: 297-316. 